A. A. Raskovalov

The Institute of High-Temperature Electrochemistry, Ural Branch of the Russian Academy of Sciences, 20 Akademicheskaya St., Yekaterinburg, 620137, Russian Federation e-mail: other@e1.ru

\title{
Simulation of borosilicate glasses with non-constant force field molecular dynamics
}

In this study the simulation of microscopical behavior of borosilicate glasses was conducted with non-constant force field molecular dynamics. The suggested model consists of classical pair potentials in the Buckingham form, long range Coulomb interaction, intramolecular bonded interactions and possibility of bond breaking and formation. The latter effects are accompanied by changes in the types of the bond-forming particles. The simulated system corresponds to the structure of borosilicate glasses with predominantly four-coordinated boron atoms. Different structure groups are formed due to the dissociation/formation of intramolecular bonds, and the processes of the glass network rearrangement intensifies with temperature increasing.

Keywords: molecular dynamics; non-constant force field; glass; borate; silicate.

Received: 11.12.2018. Accepted: 28.12.2018. Published: 29.03.2019.

(C) Raskovalov A. A., 2019

\section{Introduction}

Borosilicate glasses are materials with a wide range of applications. They can be used for nuclear waste utilization [1], as implants for tissue repair [2], in electrochemical devices as ionic conductors [3-5], as sealants for solid oxide fuel cells [6] and in many other areas of human activity. From this point of view, microscopical behavior of these glasses comes to researchers' attention. Glasses consisting of several network former oxides $\left(\mathrm{B}_{2} \mathrm{O}_{3}\right.$, $\mathrm{SiO}_{2}, \mathrm{P}_{2} \mathrm{O}_{5}$ and some others) can contain a colossal amount of possible structure groups and their combinations. For this reason numerical simulations of mixed glasses are very promoting because it can "view" glass structure at molecular level. To date, a molecular dynamics (MD) simula- tion of silicate, borate and mixed borosilicate glasses was conducted in series of papers [1-5, 7-19]. Most of these works deal with classical MD, except researches [2, 17] performed with Car-Parinello (MD) [20] and reax force field [21] (ReaxFF), correspondingly. The Car-Parinello method brings quantum mechanical calculation into MD simulation. The ReaxFF is constucted to provide chemical reactivity during MD simulation. The last two methods are used to include effects of chemical bonds dissociation and recombination to the simulation. These effects are needed to be considered for correct simulation of glass behavior. To perform such calculations with classical MD is an ambiguous and complicated, if not impossible, task. 
In the present paper I demonstrate how one can perform MD simulation of borosilicate glass with non-constant force field methodology. This approximation consists of possibility of bond breaking and formation (including changing in particle types of bond forming atoms) runtime.

\section{Simulation details}

MD simulations were performed with an original program written in $\mathrm{C}$ in canonical (NVT) ensemble. Newton's equations of motion were integrated by Velocity Verlet algorithm [22] with timestep of $0.5 \mathrm{fs}$ during 100 '000 steps. Equilibration time was $0.5 \mathrm{ps}$ (1000 timesteps). Electrostatic interactions were accounted using the Ewald summation. Nosé-Hoover thermostat [23] with relaxation time of 0.02 ps was used for maintaining the temperature around desired temperature. There was tested three temperatures: 298,500 and $1000 \mathrm{~K}$. The considered system consists of $94 \mathrm{Si}$ atoms, $80 \mathrm{~B}$ atoms, $429 \mathrm{O}$ atoms and 242 alkaline metal $(\mathrm{Me}$ ) atoms (the total number of particles is 845$)$. The oxygen atoms were divided into bridging $\left(\mathrm{O}_{c}\right)$ and non-bridging $\left(\mathrm{O}_{\mathrm{t}}\right)$ species. Bridging oxygen connects with two atoms of glass-forming element (Si/B) and non-bridging - with only one. Initial configuration of the system was generated with own special code. The box was cubic with the edge length of $20.4 \AA$ to match an approximate density of glassy silicates. Van der Waals interactions in the system were given by Buckingham pair potential:

$$
U_{i j}=A_{i j} \exp \left(-\frac{r_{i j}}{\rho_{i j}}\right)-\frac{C_{i j}}{r_{i j}^{6}},
$$

where $U_{i j}$ is the potential energy between the $i$-th and the $j$-th atoms, $r_{i j}$ is the distance between them, and $A_{i j}, \rho_{i j}$ and $C_{i j}$ are empirical parameters. Besides the Van der Waals short-range interactions, covalent bonds between oxygen and silicon/boron were set in the form of an intramolecular harmonic potential:

$$
U_{i j}=\frac{k_{i j}}{2}\left(r_{i j}-r_{0 i j}\right)^{2},
$$

where $k_{i j}$ is the spring constant and $r_{0 i j}$ is the equilibrium distance between particles. Unlike interactions provided by Eq. (1), this potential is applied only to covalent bonded pairs $\left(\mathrm{S}-\mathrm{O}_{\mathrm{t}}, \mathrm{Si}-\mathrm{O}_{c}, \mathrm{~B}-\mathrm{O}_{\mathrm{t}}\right.$ and $\mathrm{B}-\mathrm{O}_{c}$ ) listed separately. In addition, a three-body potential energy term was applied to maintain a feasible valent angle for $\mathrm{Si}-\mathrm{O}_{\mathrm{c}}-\mathrm{Si}, \mathrm{B}-\mathrm{O}_{\mathrm{c}}-\mathrm{B}$ and $\mathrm{Si}-\mathrm{O}_{\mathrm{c}}-\mathrm{B}$ bonds:

$$
U_{i j k}=\frac{k_{t b}}{2}\left(\cos \theta_{i j k}-\cos \theta_{0}\right)^{2},
$$

where $k_{t b}$ is the spring constant of the threebody potential, $100.0 \mathrm{eV}, \theta_{i j k}$ is the angle between $i j$ and $i k$ bonds, $\theta_{0}$ is the equilibrium angle, $148.3^{\circ}$ (taken from [15] for $\mathrm{Si}-\mathrm{O}-\mathrm{Si}$ angle); $i, j$ and $k$ are the indexes of $\mathrm{O}_{c}$ atom and its covalent-bonded neighbors, correspondingly.

The values of $A_{i j}, \rho_{i j}$ and $C_{i j}$ were taken from papers $[1,11,12,15,19]$. Simulations were performed with different combination of these parameters. The more suitable for the glass structure description set of the parameters is summarized in Table 1 with corresponding references. The parameters of the valent bond potential (2) are given in Table 2. The atomic charges were suggested to be found as $4 \delta, 3 \delta, a,-2 \delta$ and $-\delta$ - $a$ for $\mathrm{Si}, \mathrm{B}, \mathrm{Me}, \mathrm{O}_{\mathrm{c}}$ and $\mathrm{O}_{\mathrm{t}}$, correspondingly. The values of $\delta$ and $a$ are set to $0.4 e$ and 1.0e. Our MD program allows deleting 
The Buckingham pair potential parameters used in this study

\begin{tabular}{c|c|c|c|c}
\hline pair & $A, \mathrm{eV}$ & $\rho, \AA$ & $C, \mathrm{eV}^{6}$ & Reference \\
\hline $\mathrm{Si}-\mathrm{O}_{\mathrm{t}} / \mathrm{O}_{\mathrm{c}}$ & 13702.905 & 0.193817 & 54.681 & {$[19]$} \\
\hline $\mathrm{B}-\mathrm{O}_{\mathrm{t}} / \mathrm{O}_{\mathrm{c}}$ & 206941.81 & 0.124 & 35.0018 & {$[19]$} \\
\hline $\mathrm{Me}-\mathrm{O}_{\mathrm{t}} / \mathrm{O}_{\mathrm{c}}$ & 4383.7555 & 0.243838 & 30.700 & {$[5]$} \\
\hline $\mathrm{O}_{\mathrm{t}} / \mathrm{O}_{\mathrm{c}}-\mathrm{O}_{\mathrm{t}} / \mathrm{O}_{\mathrm{c}}$ & 352.56 & 0.35 & 0 & {$[1]$} \\
\hline $\mathrm{Si}-\mathrm{B}$ & 337.584 & 0.29 & 0 & {$[1]$} \\
\hline $\mathrm{Si}-\mathrm{Me}$ & 861.744 & 0.29 & 0 & {$[1]$} \\
\hline $\mathrm{Si}-\mathrm{Si}$ & 836.16 & 0.29 & 0 & {$[1]$} \\
\hline $\mathrm{B}-\mathrm{B}$ & 121.056 & 0.29 & 0 & {$[1]$} \\
\hline $\mathrm{Me}-\mathrm{Me}$ & 9500.0 & 0.23 & 0 & {$[12]$}
\end{tabular}

(adding) valent bonds from (to) the corresponding list to mimic processes of bonds dissociation and glass forming:

$$
\ldots \equiv \mathrm{Si}-\mathrm{O}_{\mathrm{c}}-\mathrm{R} \leftrightarrow \equiv \mathrm{Si}+\mathrm{O}_{\mathrm{t}}-\mathrm{R}
$$

and

$$
\ldots \equiv \mathrm{B}-\mathrm{O}_{\mathrm{c}}-\mathrm{R} \leftrightarrow-\mathrm{B}+\mathrm{O}_{\mathrm{t}}-\mathrm{R} .
$$

If the bonds automatically break (form) then the interatomic distance is higher (lower) than a maximal bond distance parameter $\left(r_{m}\right)$. The values of this parameter are given in Table 2. The further dissocia- tion with participation of $\mathrm{Si}+$ or $\mathrm{B}+$ species is not allowed. The charges of $\mathrm{Si}+$ and $\mathrm{B}+$ species are chosen to keep electroneutrality during reactions (4) and (5).

Table 2

Valent bonds parameters

(including the maximal bond distance, $\mathrm{rm}$ ) used in this study

\begin{tabular}{c|c|c|c}
\hline pair & $k, \mathrm{eV}$ & $r_{0}, \AA$ & $r_{m}, \AA$ \\
\hline $\mathrm{Si}-\mathrm{O}_{\mathrm{t}}$ & $10^{-4}$ & 1.625 & - \\
\hline $\mathrm{Si}-\mathrm{O}_{\mathrm{c}}$ & $10^{-4}$ & 1.650 & 2.0 \\
\hline $\mathrm{B}-\mathrm{O}_{\mathrm{t}}$ & $10^{-5}$ & 1.400 & - \\
\hline $\mathrm{B}-\mathrm{O}_{\mathrm{c}}$ & $10^{-5}$ & 1.430 & 1.9
\end{tabular}

\section{Results and Discussion}

The obtained structure of the system is presented in Fig. 1. One can see that the simulated glass consists of branchy chains of $\mathrm{Si}-\mathrm{O} / \mathrm{B}-\mathrm{O}$ bonds which can form various combinations and loops. In principle, there are two possible boron coordinations in borate glasses, triangular and tetrahedral [24]. In our case almost all boron in the glass is four-coordinated, Fig. 1.

Radial distribution functions (RDFs) are presented in Fig. 2. All RDF-curves consist of one sharp and several broad maximums. This is typical of liquids or amorphous systems and indicates the

presence of the short-range and the absence of the long-range orders. There are no series of well-resolved maxima, specific for crystalline solids. The first maximum of RDF-curve corresponds to the most probable distance in the near-neighbor coordination. According to Fig 2(a), the distance between $\mathrm{Me}$ and $\mathrm{O}_{\mathrm{t}}$ is less than that between $\mathrm{Me}$ and $\mathrm{O}_{c}$. It can be explained by more negative charge of $\mathrm{O}_{t}$ species (Simulation Details section) and, therefore, stronger Coulomb interaction. RDFs almost do not depend on temperature, the differences are observed only for the RDFs 
with the sharpest maxima, for example, $\mathrm{Si}-\mathrm{O}$. For these RDFs the value of the first maximum decreases with temperature increasing and the peak becomes broader, Fig. 2(b). This process occurs, most likely, due to the thermal motion. The first maximum for $\mathrm{B}-\mathrm{O}_{\mathrm{t}} / \mathrm{O}_{\mathrm{c}}$ and $\mathrm{Si}-\mathrm{O}_{\mathrm{t}} / \mathrm{O}_{\mathrm{c}}$ pairs locates at distance of 1.375-1.425 and 1.575-1.675 ̊̊, correspondingly, which

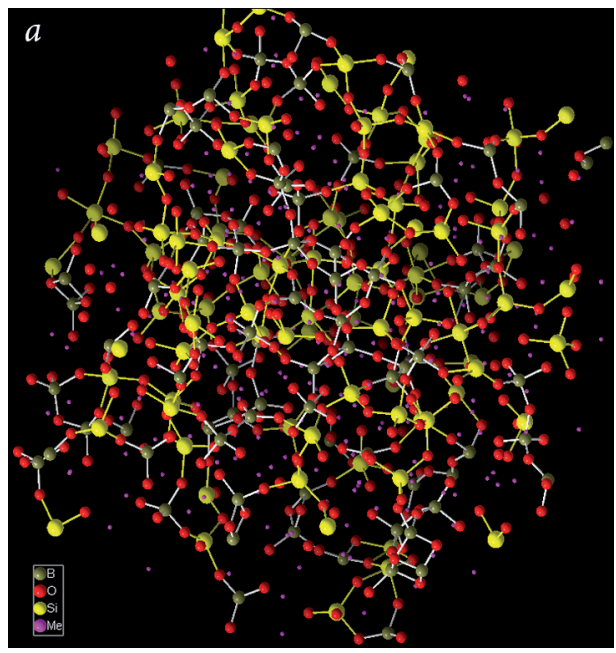

is close to $\mathrm{B}-\mathrm{O}$ и $\mathrm{Si}-\mathrm{O}$ interatomic distances in borosilicate glasses [15].

As the system initially does not have crystalline lattice and a corresponding look of RDF-curves, it is necessary to find another criterion of transition between liquid and solid states. Such a criterion can be, for example, mean square displacement (MSD), which characterizes

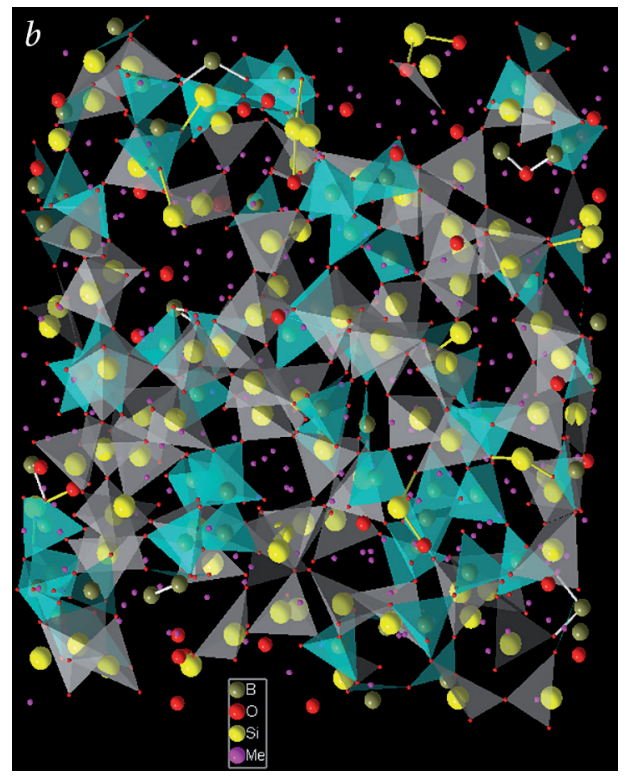

Fig. 1. The structure of the simulated system after 30'000 timesteps at $298 \mathrm{~K}$ in (a) stick-and-ball and (b) polyhedral representation.

Blue polyhedra correspond to boron central atom, gray - to silicon

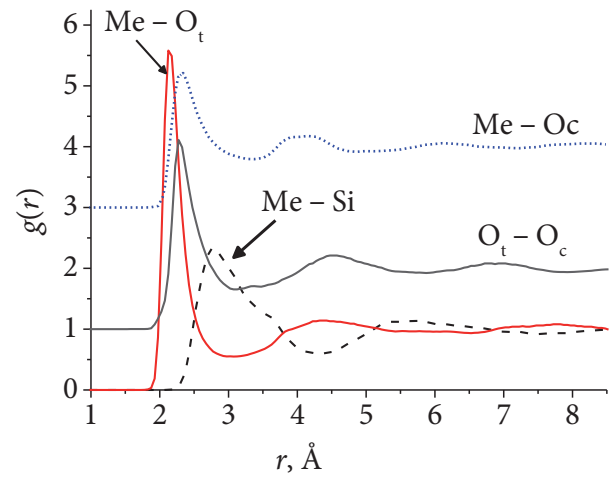

$a$

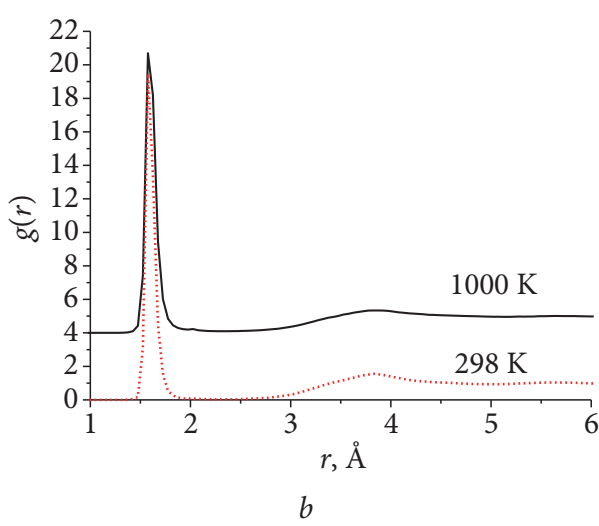

Fig. 2. Some radial distribution functions, $g(r)$ of the simulated glass:

(a) for $298 \mathrm{~K}$; (b) for pair $\mathrm{Si}-\mathrm{O}_{\mathrm{t}}$ at 298 and $1000 \mathrm{~K}$ 
a mobility of ions. In the simulated borosilicate glass, MSDs of most ions tend to reach a plateau with some oscillation at temperature of $298 \mathrm{~K}$, except one for $\mathrm{Me}$ ions increased linearly, Fig. 3(a). The time derivative of MSD grows with temperature increasing, Fig. 3(b). This means that all species start to diffuse and the system behavior becomes liquid-like. Analogous effects can be seen on trajectories of the ion motion. At temperature of $298 \mathrm{~K}$ most ions vibrate around one point (although with big enough amplitude), Fig. 4(a). An exception is the Me ion, its trajectory consists of a series of hops between positions

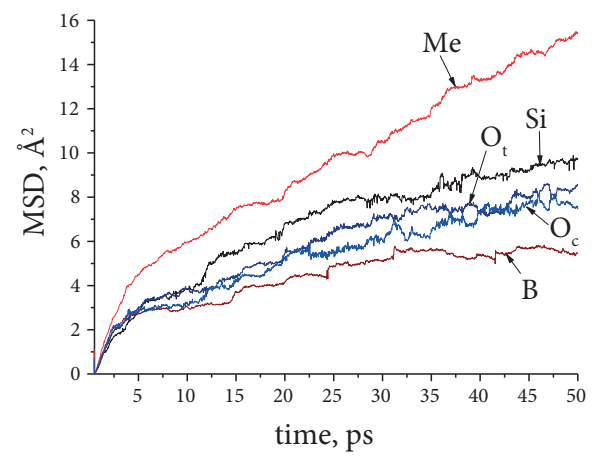

$a$ with long enough oscillation time. With temperature increasing, Me ion spends less time in one position, more often hops and covers greater distance, Fig. 4(b). Its movement character becomes more and more liquid-like.

Fig. 5 demonstrates the number of $\mathrm{Si}$ and $B$ species as a function of time. The changes in these quantities are provided by dissociation of $\mathrm{B}-\mathrm{O}_{\mathrm{c}}$ and $\mathrm{Si}-\mathrm{O}_{\mathrm{c}}$ bonds according to equations (4) and (5). Since dissociation in the suggested model occurs upon reaching the determined distance (Table 2), the observed fluctuations of the particles numbers are related with

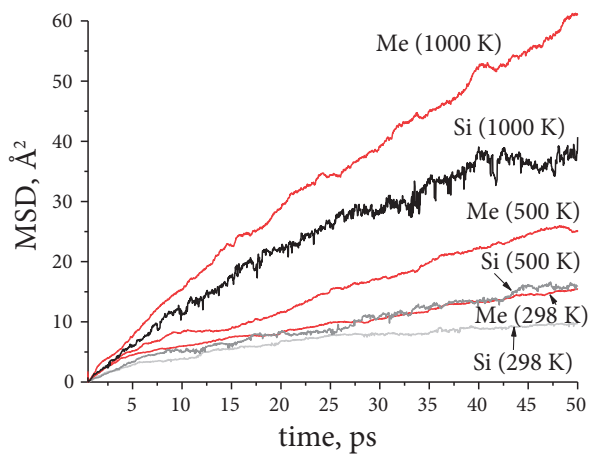

$b$

Fig. 3. The mean square displacement (MSD) of the simulated borosilicate glass:

(a) for different ions at $298 \mathrm{~K}$; $(b)$ for Me and Si ions at different temperatures

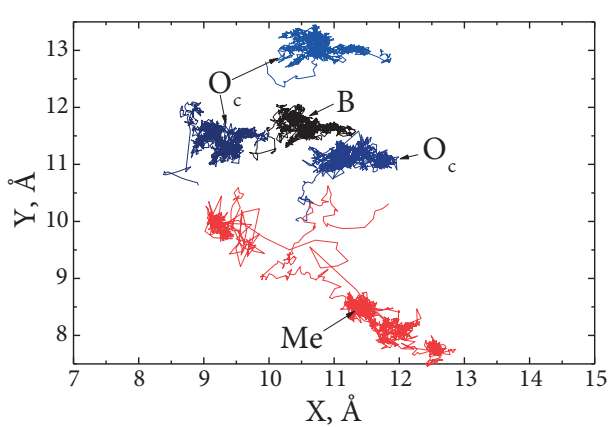

$a$

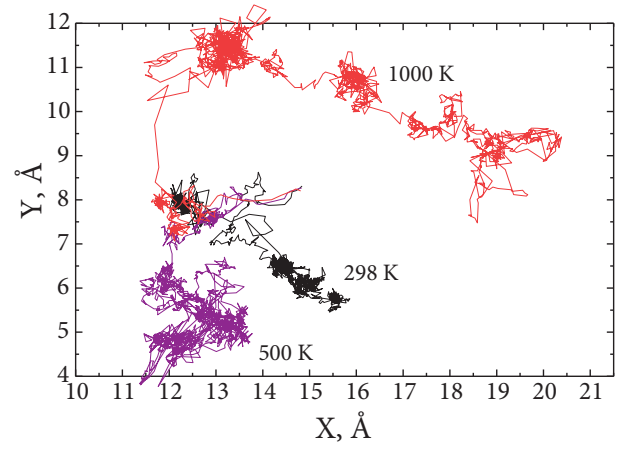

$b$

Fig. 4. Trajectories of motion in the simulated borosilicate glass for:

(a) different ions at $298 \mathrm{~K} ;(b)$ the same Me ion at different temperatures 
changing in the $\mathrm{B} / \mathrm{Si}-\mathrm{O}_{\mathrm{c}}$ distances. According to Fig. 5, the suggested pair potentials provide stronger $\mathrm{B}-\mathrm{O}_{\mathrm{c}}$ bonding, then $\mathrm{Si}-\mathrm{O}_{\mathrm{c}}$ one, as the amplitude of the $\mathrm{Si}$ number oscillation is bigger. Also, Fig. 5 demonstrates that the amplitudes of these oscillations increase with temperature. This is a result of thermal motion which promotes overcoming of forces of interatomic attraction, as noted above in the discussion of the radial distribution functions. The bonds breaking and formation realized by methodology of non-constant force field allow glass structure to be "dynamical," i.e. to destroy ones atomic groups and to cre- ate others. A variety of formed molecular groups can see in Fig. 1.

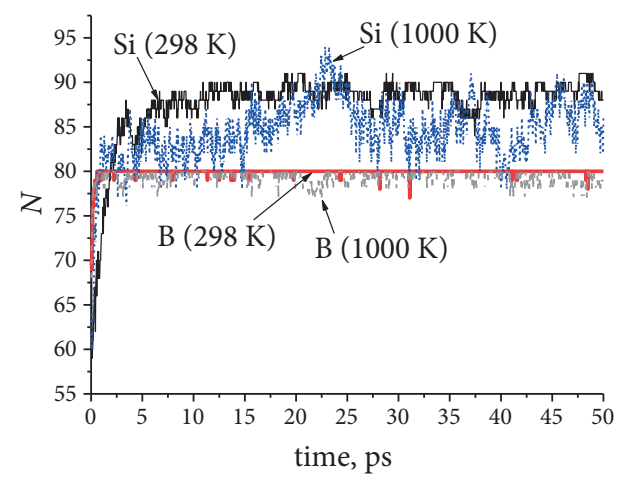

Fig. 5. The number of Si and B species as a function of simulation time

\section{Conclusions}

In the paper reference classic pair potentials have been tested for molecular dynamic simulation of borosilicate glasses. The suggested combination of the potentials and ionic charges describes interatomic distances in borosilicate glasses reasonably well. Oxygen atoms were divided into bridging and non-bridging by the value of electrical charge. The transition between these states is possible due to bond formation/dissociation. These phenomena are implemented by non-constant force field molecular dynamics. It has been shown that the degree of the bond dissociation increases with temperature growth.

\section{Acknowledgments}

The reported study was funded by Russian Foundation for Basic Research (RFBR), according to the research project No. 16-33-60095 mol_a_dk.

\section{References}

1. Delaye JM, Ghaleb D. Molecular dynamics simulation of a nuclear waste glass matrix. Materials Science and Engineering B. 1996;37:232-6.

2. Tilocca A. Sodium migration pathways in multicomponent silicate glasses: Car Parrinello molecular dynamics simulations. J Chem Phys. 2010;133:014701. DOI:10.1063/1.3456712.

3. Verhoef AH, Den Hartog HW. Molecular Dynamics simulations of borate glasses. Rad Effect Defect Solids. 1991;119-121(2):493-8.

DOI:10.1080/10420159108220770.

4. Varsamis C-PE, Vegiri A, Kamitsos EI. Molecular dynamics investigation of lithium borate glasses: Local structure and ion dynamics. Phys Rev B. 2002;65:104203. DOI:10.1103/PhysRevB.65.104203. 
5. Cormack AN, Du J, Zeitler TR. Alkali ion migration mechanisms in silicate glasses probed by molecular dynamics simulations. Phys Chem Chem Phys. 2002;4:3193-7. DOI:10.1039/b201721k.

6. Smeacetto F, Miranda A, Chrysanthou A, et al. Novel Glass-Ceramic Composition as Sealant for SOFCs. J Am Ceram Soc. 2014;97:3835-42. DOI:10.1111/jace.13219.

7. Inoue H, Aoki N, Yasui I. Molecular Dynamics Simulation of the Structure of Borate Glasses. J Am Ceram Soc. 1987; 70(9):622-7.

DOI:10.1111/j.1151-2916.1987.tb05729.x.

8. Xu Q, Kawamura K, Yokokawa T. Molecular dynamics calculations for boron oxide and sodium borate glasses. J Non-Cryst Solids. 1988;104(2-3):261-72. DOI:10.1016/0022-3093(88)90397-3.

9. Vessal B, Amini M, Leslie M, Catlow CRA. Potentials for Molecular Dynamics Simulation of Silicate Glasses. Mol Sim. 1990;5(1-2):1-7. DOI:10.1080/08927029008022407.

10. Cormack AN, Cao Yu. Molecular Dynamics Simulation of Silicate Glasses. Mol Eng. 1996;6:183. DOI:10.1007/BF00161727.

11. Rossano S, Ramos A, Delaye J-M, Creux S, Filipponi A, Brouder Ch, Calas G. EXAFS and Molecular Dynamics combined study of $\mathrm{CaO}-\mathrm{FeO}-2 \mathrm{SiO}_{2}$ glass. New insight into site significance in silicate glasses. Europhys Lett. 2000;49(5):597-602.

12. Cormak AN, Du J. Molecular dynamics simulation of soda-lime-silicate glasses. J Non-Cryst Solids. 2001;293-295:283-9.

13. Gou F, Greaves GN, Smith W, Winter R. Molecular dynamics simulation of sodium borosilicate glasses. J Non-Cryst Solids. 2001;293(1):539-46.

DOI:10.1016/S0022-3093(01)00775-X.

14. Sawaguchi N, Yamaguchi K, Sasaki M, Kawamura K. Interatomic Potential Model for Molecular Dynamics Simulation of Lithium Borate Melts/Glasses. J Comp Chem. 2015;14(4):139-46. DOI:10.2477/jccj.2015-0017.

15. Scherer C. Molecular dynamics simulations of silicate and borate glasses and melts: Structure, diffusion dynamics and vibrational properties [dissertation]. Mainz (Germany): Fachbereich Physik, Mathematik und Informatik der Johannes GutenbergUniversitat; 2015. 189 p.

16. Li X, Song W, Yang K, Anoop Krishnan NM, Wang B, Smedskjaer MM, Mauro JC, Sant G, Balonis M, Bauchy M. Cooling rate effects in sodium silicate glasses: Bridging the gap between molecular dynamics simulations and experiments. J Chem Phys. 2017; 147:074501. DOI:10.1063/1.4998611.

17. Yu Y, Wang B, Wang M, Sant G, Bauchy M. Reactive molecular dynamics simulations of sodium silicate glasses - Toward an improved understanding of the structure. Int J Appl Glass Sci. 2017;8:276-84. DOI:10.1111/ijag.12248.

18. Stevensson B, Yu Ya, Ed'en M. Structure-Composition Trends in Multicomponent Borosilicate-Based Glasses Deduced from Molecular Dynamics Simulations with Improved B - O and P - O Force Fields. Phys Chem Chem Phys. 2018;20:8192-209. DOI:10.1039/C7CP08593A.

19. Wang M, Anoop Krishnana NM, Wang B, Smedskjaer MM, Mauro JC, Bauchya M. A new transferable interatomic potential for molecular dynamics simulations 
of borosilicate glasses. J Non-Cryst Solids. 2018;498:294-304.

DOI:10.1016/j.jnoncrysol.2018.04.063.

20. Car R, Parrinello M. Unified Approach for Molecular Dynamics and Density-Functional Theory. Phys Rev Lett. 1985;55:2471-4. DOI:10.1103/PhysRevLett.55.2471.

21. van Duin ACT, Dasgupta S, Lorant F, Goddard WA. Reax FF: A Reactive Force Field for Hydrocarbons. J Phys Chem A. 2001;105:9396-409. DOI:10.1021/jp004368u.

22. Verlet L. Computer "Experiments" on Classical Fluids. I. Thermodynamical Properties of Lennard-Jones Molecules. Phys Rev. 1967;159(1):98-103.

DOI:10.1103/PhysRev.159.98.

23. Martyna GJ, Tuckerman ME, Tobias DJ, Klein ML. Explicit Reversible Integrators for Extended Systems Dynamics. Mol Phys. 1996;87(5):1117-57. DOI:10.1080/00268979600100761.

24. Wu J, Stebbins JF. Cation Field Strength Effects on Boron Coordination in Binary Borate Glasses. J Amer Ceram Soc. 2014; 97(9):2794-801.

DOI:10.1111/jace.13100. 